\title{
The Functional Outcome of Arthroscopic Anterior Cruciate Ligament Reconstruction in Patients Using Different Graft Tension During Tibial Fixation
}

\author{
Gabriel Alfonso B. Javier, M.D.' \\ Alberto Ma. V. Molano, M.D. ${ }^{2}$
}

\begin{abstract}
Background Anterior Cruciate Ligament ( $\mathrm{ACL}$ ) reconstruction is commonly performed to restore knee kinematics and halt the progression of osteoarthritis. A primary variable that could influence the outcome of $\mathrm{ACL}$ reconstruction is the tension applied to the graft at the time of fixation. If the tension is too great, an abnormal compressive force could potentially develop across the tibiofemoral joint, hindering knee motion, and subjecting the articular surfaces to increased stress. If the tension in the graft is too low, the graft will not be effective in restoring normal kinematics. The Tegner Lysholm Knee Scale is a functional scoring for patients with ligamentous injuries. It is a patient-reported measure of knee function and is important for comprehensive assessment conditions in both the clinical and research context. Our objective was to compare which tension technique $(15 \mathrm{lbs}$
\end{abstract}

Gabriel B. Javier, MD

gbjavier_2003@yahoo.com

Department of Orthopaedics, University of Santo Tomas Hospital

2 Department of Orthopaedics, University of Santo Tomas Hospital graft tension using a Mitek Tensioner ${ }^{\mathrm{TM}}$ vs maximal sustained two-hand technique) would yield better functional outcome at 6 months and 12 months postoperatively using the Tegner Lysholm Knee Scale.

Methods Twenty-nine patients who underwent arthroscopic $\mathrm{ACL}$ reconstruction at the University of Santo Tomas Hospital Private Division were randomly divided equally into two groups (group $A$ or group B). During tibial fixation, group A would receive 15 lbs graft tension using a Mitek Tensioner and group $B$ would receive graft tension using the maximal sustained two-handed pull technique. The patients underwent a standard rehabilitation protocol at an institution of their choice and a Lysholm Scoring Scale and Tegner activity scale were self-administered at 6 months and 12 months after the surgery in order to assess their functional outcome.

Results The results showed that the functional outcome scores of group A were higher than group B. The yielded p-value was 0.10 (6 months), 0.07 (12 months) for group $A$ and 0.27 (6 months), 0.46 (12 months) for group $B$. The results showed no sufficient evidence of a significant difference between the effects of arthroscopic $A C L$ reconstruction with $15 \mathrm{lbs}$ weight using a Mitek Tensioner (group A) and graft tension using the maximal sustained two-handed pull 
technique (group B) in the knee functional outcome of patients at 6 months and 12 months postoperatively. Conclusion: The functional outcome scores of patients who underwent $A C L$ reconstruction using different graft tension did not show significant results. Further re-evaluation of patients' functional outcome score is necessary after 12 months postoperatively. The desired tensioning technique of the $\mathrm{ACL}$ surgeon would be at his/her convenience knowing beforehand the pros and cons of each technique.

Keywords: Arthroscopic ACL reconstruction, Lysholm Scoring Scale, Tegner activity scale, graft tension, tibial fixation

\section{INTRODUCTION}

The anterior cruciate ligament $(\mathrm{ACL})$ is one of the most frequently injured structures in the knee joint. Since the ACL fails to heal biologically, operative and reconstructed techniques have been emphasized for patients who desire the restoration of knee function and stability as well as a return to high-level physical performance.[1]

The tension applied to the $A C L$ graft during tibial fixation is thought to influence graft healing, knee kinematics, and joint contact forces. There is still a controversy about the optimal amount of tension and tension technique applied to the graft as well as the best fixation device to use. Moreover, the operating surgeon must exercise considerable judgement when applying these theories and techniques. If the graft is too loose, the knee will be unstable. If it is too tight, then the graft will be under strain and may break. Proper graft tensioning is also deemed to be important for restoring normal anteroposterior laxity in $\mathrm{ACL}$ reconstruction during graft fixation. The current clinical guidelines and practice seem to follow the theories proposed by laboratory-based studies that the average initial graft tension for hamstring tendon grafts used by surgeons is $70 \mathrm{~N}$ (15 lbs). This remains a controversial area and many surgeons prefer to tension $\mathrm{ACL}$ grafts using manual feedback or manual pull in order to determine the amount of tension applied to the graft at the time of fixation. This technique, referred to as the unmeasured initial tension or the maximal sustained two-handed pull technique, is currently the commonly used tensioning technique or protocol.[2] This technique is defined as the maximum force that could be sustained by the operating surgeon for a 1- to 2-minute period. However, the maximal sustained two-handed pull technique is difficult to reproduce from trial-to-trial and would be different between surgeons since several factors can affect its reproducibility, such as strength and age of the surgeon and even the timing of the operation, especially when the surgeon would do several operations and fatigue sets in.

Several commercially available tensioning devices (Mitek Tensioner) can reportedly produce and maintain equal tension in the strands of hamstring tendon graft. In principle, when the tensioning device is pulled, it exerts equal tension in all of the hamstring graft strands. However, when the tensioning device deviates from its midline axis, which may occur while driving an interference screw, strand tension may alter. This can have an adverse effect on the biomechanical properties of the graft, which may then affect the surgical outcome. The goal of all the tensioning methods is clear and that is to restore anterior and anatomic stability to the knee without risking graft failure or complications created by poor surgical technique.[3]

The next step after arthroscopic ACL reconstruction is the rehabilitation and return of the patient to physical activity. Rehabilitation plays a vital role in getting the patients back to their activities. A physical therapy program helps the patient regain knee strength and range of motion. The final phase of rehabilitation is aimed at a functional return tailored to the athlete's sport with additional strength and conditioning in order to avoid another potential injury in the future.

Patient-reported measures of functional outcomes of the knee are important for comprehensive assessment conditions in both clinical and research contexts. Important parameters include pain, function, quality of life, and activity level. Patient-directed activity level scales complement existing outcome instruments by projecting a measure of "what the patients can and cannot do". One of the widely used activity level scales, the Tegner activity scale was designed as a score for the activity level to complement the functional Lysholm knee score for patients with ligamentous injuries and sprains. The Tegner Lysholm Knee Scale was first published in 1982 and later revised in 1985, which led to the removal of several items such as references to walking and running while emphasizing the parameters regarding instability, pain, and swelling.[4] 
The Lysholm scale uses basic and simple language in its questionnaire form. It is one of the most widely used tools for research and in the clinical context on ligamentous injuries, meniscal injuries, chondral defect, and recurrent patellar dislocations. The Tegner activity scale has been the most widely used self-reported rating scale for preoperative and postoperative knee surgeries. It is very simple and cost-effective and has widespread use as a patient-reported activity scoring system for patients with various knee conditions. [5]

The study aims to compare the functional outcome between the use of a $15 \mathrm{lbs}$ graft tension with the Mitek tensioner versus the maximal sustained twohand technique, measured using the Lysholm Knee Scoring Scale and the Tegner activity scale at 6 months and 12 months post $A C L$ reconstruction.

\section{METHODOLOGY}

Twenty-nine patients with unilateral $A C L$ tear who underwent $A C L$ reconstruction at the UST Hospital Private Division were divided into two separate groups, either A or B using simple randomization via "coin toss." Group A received 15 lbs weight using a Mitek Tensioner and group B received tension during fixation using the maximal sustained two-handed pull technique. The patients underwent arthroscopic $\mathrm{ACL}$ reconstruction using a single bundle hamstring graft. After creating the femoral and tibial tunnels, the hamstring graft fixation was done using a bioabsorbable screw. The amount of tension during fixation of the hamstring graft would either be $15 \mathrm{lbs}$ weight using a Mitek Tensioner (group A) or the maximal sustained two-handed pull technique (group B). The loads were applied to the graft at 30 degrees of knee flexion. Knee immobilizer was also applied postoperatively. The patients underwent a standard rehabilitation protocol at an institution of their choice. The protocol was divided into two parts: The first part was the early rehabilitation, which focused on regaining range of motion, normalizing gait, and activation of quadriceps muscles. The latter part of the rehabilitation was at the 4- to 6-month mark postoperatively and involves plyometric training, agility drills, instructions for takeoff and landing mechanics and functional testing. The Lysholm Knee Scoring Scale and the Tegner activity scale (questionnaire) were self-administered among all the patients at 6 months and 12 months after the surgery. Both questionnaires are self-assessment tools that evaluate the functional outcome scores of the patients. A two-sample independent t-test was used to evaluate the data gathered.

\section{Ethical Considerations}

Strict privacy and confidentiality were employed in our study. The patients were given informed consent prior to the operation. It includes information about the procedure, duration, side effects and risks, benefits, confidentiality, and data sharing. The patients who voluntarily gave their consent were included in our study.

\section{RESULTS}

Twenty-nine patients with complete $A C L$ tear who underwent arthroscopic $\mathrm{ACL}$ reconstruction at the UST Hospital from December 2014 to January 2016 were included in the study. The age range of the patients was between 17 and 51 years old with a mean age of 25 . Only two females were part of our study and the rest were males. Most of them reported sports-related injury as the cause of their complete $A C L$ tear attributed to either basketball or dance as the specific activity. Three patients were part of a competitive lower division sport and one patient was considered inactive with no involvement in any sport. Only two patients were able to return to before-injury level of activity. The most commonly reported symptoms after 6 months were pain and swelling of affected limbs. By 12 months post surgery, the patients did not report any symptoms. There were no drop-outs in the study.

At 6 months and 12 months postoperatively, the p-value of the Lysholm Knee Scores was 0.10 and 0.07 . Hence there was no sufficient evidence to show a significant difference between the effects of arthroscopic $A C L$ reconstruction with 15 lbs weight using a Mitek Tensioner (group A) and graft tension during tibial fixation using the maximal sustained two-handed pull technique (group B) in the Lysholm Knee Scoring Scale of patients after 6 months and 12 months postoperatively. Although the difference of scores between the two groups was not significant, it was noted that group $A$ patients had higher mean functional outcome scores after 6 months and 12 months.

At 6 months and 12 months postoperatively, the $p$-values of the Tegner activity scale were 0.27 and 0.46 . Again, there was no sufficient evidence to conclude a significant difference between the effects of arthroscopic $A C L$ reconstruction with 15 lbs 


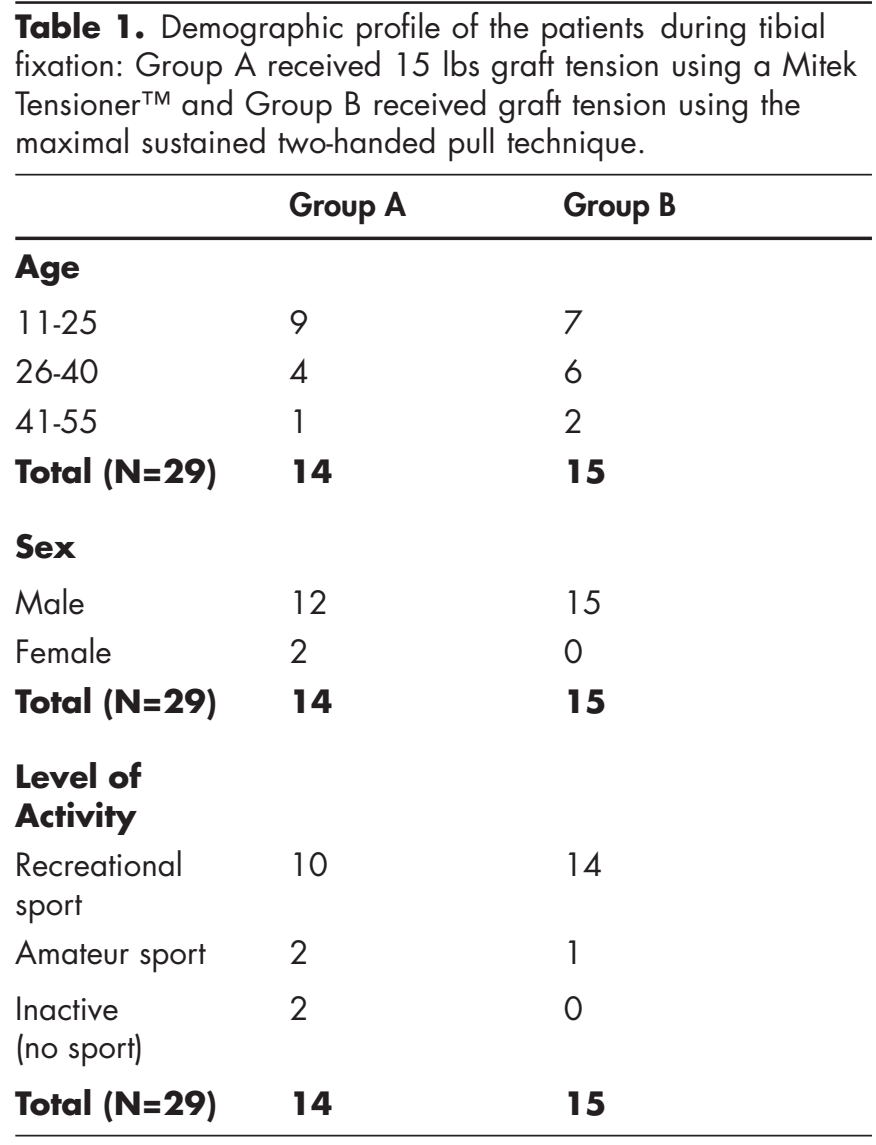

weight using a Mitek Tensioner (group A) and graft tension during tibial fixation using the maximal sustained two-handed pull technique (group $B$ ) in the Tegner activity scale of patients after 6 months and 12 months postoperatively. But while the difference is not statistically significant, it can be noted, however, that group A patients had higher mean functional outcome scores after 6 months and 12 months.

\section{DISCUSSION}

The tension applied to the ACL graft before graft fixation is significantly influenced by the kinematics of the knee joint and its ability to consolidate inside the joint. A low-intensity graft tension will not provide adequate joint stability, while high-intensity graft tension will permit a functional range of motion but is prone to early graft failure or re-rupture.[5]

The maximal sustained two-handed pull technique relies on feedback from the graft to the operating surgeon and his/her interpretation of the feedback. It is influenced by several factors such as the length and diameter of the graft material, the size and length of the bone tunnels, the quality of the bone and the graft material being used, and by the accuracy of tunnel placement. These factors are dependent on the expertise of the surgeon, but some factors are patient factors that cannot be controlled by the operating surgeon. [6] Because of this, a theory has been brought up that even an experienced operating sports surgeon would be unable to exactly re-create the amount of sustained pull and tension applied to the graft and with different mean tensions to the graft across all operating surgeons.

Some sports surgeons prefer to use a tensioning device because the amount of tension can be dialed-in. The logic behind this is the joint reaction forces from the tensioning device improve anterior knee stability. [7] It can produce the desired amount of tension depending on the pull of the surgeon and maintain equal tension of the $\mathrm{ACL}$ graft strands after looping it around the tensioner during fixation.

The outcomes following $A C L$ reconstruction may be determined from subjective (Lysholm and Tegner) and objective clinical scores (IKDC and KOOS). [8] In many clinical research studies, these questionnaires are used as functional outcome measures because they accurately and reliably reflect symptoms, complaints, and disabilities that are specific and important to patients. [9] Clinical research studies have placed a great deal of emphasis on the perspective

Table 2. Functional outcome results between the two groups

\begin{tabular}{|c|c|c|c|c|c|}
\hline & \multirow[b]{2}{*}{$\begin{array}{l}\text { Number of } \\
\text { participants }\end{array}$} & \multicolumn{2}{|c|}{ Lysholm Knee Scoring Scale (Mean +/- SD) } & \multicolumn{2}{|c|}{ Tegner Activity Scale (Mean +/- SD) } \\
\hline & & 6 months & 12 months & 6 months & 12 months \\
\hline Group A & 14 & $\begin{array}{l}84.58 \\
(+/-4.92)\end{array}$ & $\begin{array}{l}84.75 \\
(+/-3.96)\end{array}$ & $\begin{array}{l}2.58 \\
(+/-0.9)\end{array}$ & $\begin{array}{l}2.83 \\
(+/-0.68)\end{array}$ \\
\hline \multirow[t]{3}{*}{ Group B } & 15 & $\begin{array}{l}81.58 \\
(+/-3.58)\end{array}$ & $\begin{array}{l}81.50 \\
(+/-3.94)\end{array}$ & $\begin{array}{l}2.25 \\
(+/-0.15)\end{array}$ & $\begin{array}{l}2.58 \\
(+/-0.5)\end{array}$ \\
\hline & $p$ value & $>0.05$ & $>0.05$ & $>0.05$ & $>0.05$ \\
\hline & Total $(\mathbf{N}=\mathbf{2 9})$ & $p$ value (>0.05) & $p$ value (>0.05) & $p$ value (>0.05) & $p$ value $(>0.05)$ \\
\hline
\end{tabular}


of the patient with the use of quality of life instruments such as the Lysholm Knee Score and the Tegner activity scale, thus classifying these instruments as functional outcome assessment tools.

In this present study, we evaluated the functional outcomes of patients who underwent arthroscopic $\mathrm{ACL}$ reconstruction using two different tensioning methods: the use of Mitek Tensioner with $15 \mathrm{lbs}$ weight (Figure 1) and maximal sustained two-handed pull technique (Figure 2). The functional outcome scores from the Lysholm Knee Score and Tegner activity scale demonstrated acceptable parameters like consistency, validity, and responsiveness. These applied scales were not deemed to interpret the individual items for every scale but it generally provided information as to the progress of functionality on each patient who underwent $A C L$ reconstruction 6 months prior. The Tegner activity scale revealed consistent findings as expected. There is a decrease in performance with reported conditions such as pain and swelling. Moreover, several patients were unable to comply with their rehabilitation protocol, which is the reason for a re-evaluation of the patient's functional outcome score after 12 months post surgery.

\section{CONCLUSION}

While to date controversy exists regarding the amount of tension used during tibial fixation, the maximal sustained two-handed pull technique is the widely used protocol, and the use of the Mitek Tensioner is very promising for $\mathrm{ACL}$ reconstruction. In theory, the two-handed pull technique poses many problems. The amount of tension cannot be directly measured and it cannot also be sustained and reproduced from time-to-time. In addition, our results showed a lesser mean functional outcome activity scale for patients under group $B$ with the use of the maximal sustained two-handed pull technique. However, statistical analysis showed no significant difference. We recommend the continuation of this study in order to re-evaluate the functional outcome activity scale of the same groups after 12 months and beyond. By that time, there would also be a larger sample/population size for comparison.

In order to address the limitations of this present study, we recommend the following: (1) A larger sample/population size; (2) The importance of the rehabilitation protocol must be stressed out to the patients comprising the sample population; (3) The sample population should be limited to recreational and competitive athletes only; (4) The sedentary, disabled, and elderly (>65 yrs old) should be excluded in the study, and (5) Further follow-up, reassessment, and observation is recommended for greater than 12 months. 


\section{REFERENCES}

1. Roos PJ, Hull ML, Howell SM. Lengthening of double-looped tendon graft constructs in three regions after cyclic loading: a study using Roentgen stereophotogrammetric analysis. J Orthop Res. 2004;22(4):839-46.

2. Fleming B, Beynnon B, Howe J, McLeod W, Pope M. Effect of tension and placement of a prosthetic anterior cruciate ligament on the anteroposterior laxity of the knee. Journal of Orthopaedic Research. 1992 Mar; 10(2):177-86.

3. Coleridge SD, Amis AA. A comparison of five tibial-fixation systems in hamstring-graft anterior cruciate ligament reconstruction. Knee Surgery, Sports Traumatology, Arthroscopy. 2004 Sep 1;12(5):391-7.

4. Briggs KK. Lysholm score and Tegner activity level in individuals with normal knees. Am J Sports Med. 2009 May;37(5):898-901. Epub 2009 Mar 23.

5. Dargel J, Gotter M, Mader M, Pennig D. Biomechanics of the Anterior Cruciate Ligament and Implications for Surgical Reconstruction. Strategies Trauma Limb Reconstr. 2007 Apr; 2(1):1-12.

6. Oneill J, Byrne F, Hirpara K, McHugh P, Curtin W. Anterior Cruciate Ligament Graft Tensioning. Is the Maximal Sustained Pull Technique Reproducible? BMC Research Notes. 2011 July 20.

7. Thompson DM, Hull ML, Howell SM. Does a tensioning device pinned to the tibia improve knee anterior-posterior load-displacement compared to manual tensioning of the graft following anterior cruciate ligament reconstruction? A cadaveric study of two tibial fixation devices. Journal of Orthopaedic Research. 2006 Sep;24(9): 1832-41.

8. Carmont M. Anatomic Single Bundle ACL Reconstruction. Curr Rev Musculoskelet Med. 2011 June;4(2):65-72.

9. Marx RG, Jones EC, Allen AA, Altchek DW, O'Brien SJ, Rodeo SA, et al. Reliability, validity, and responsiveness of four knee outcome scales for athletic patients. JBJS. 2001 Oct 1;83(10): 1459-69.

\section{APPENDIX}

\section{Mitek Tensioner}

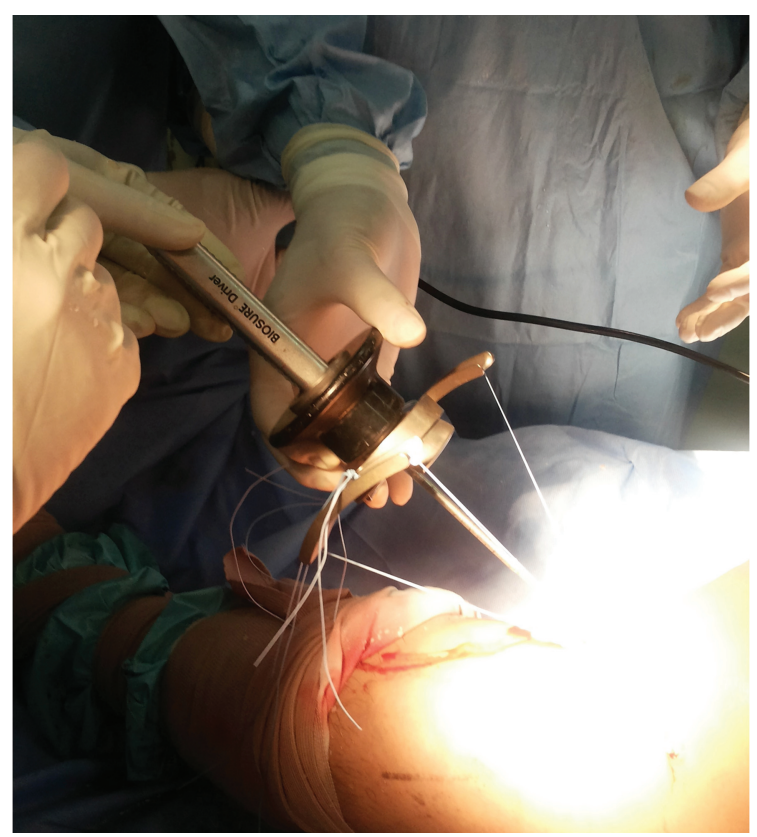

Two-handed pull technique

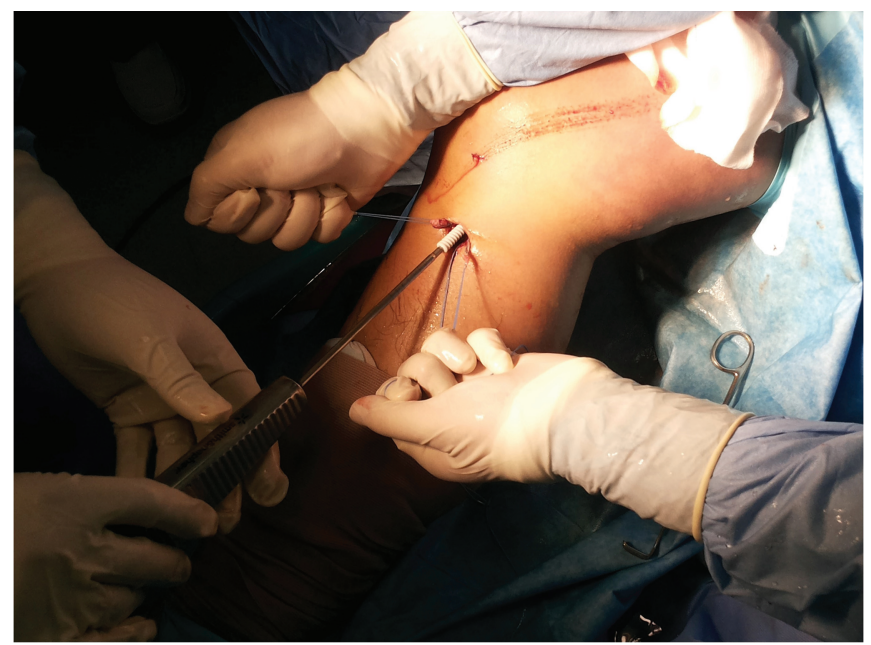

\section{TEGNER ACTIVITY SCALE}

Please indicate in the spaces below the HIGHEST level of activity that you participated in BEFORE YOUR INJURY and the highest level you are able to participate in CURRENTLY.

Before injury: Level:

Current:

Level:

Level 10 Competitive sports - soccer, football, rugby

Level 9 Competitive sports - soccer, football, rugby, wrestling, gymnastics, basketball

Level 8 Competitive sports - racquetball, squash, badminton, track and field, athletics (jumping), downhill skiing

Level 7 Competitive sports - tennis, athletics (running), motocross, handball, basketball

Recreational sports - soccer, ice hockey, squash, athletics (jumping), cross-country track

Level 6 Recreational sports - Tennis or badminton, handball or basketball, downhill skiing, jogging, at least 5 times weekly

Level 5 Competitive sports - Bicycling, cross-country skiing, recreational sports, jogging on uneven ground $>2$ times weekly

Work - heavy labor (eg, building, forestry) 
Level 4 Recreational sports - Bicycling, cross-country skiing, jogging on uneven ground $>2$ times weekly

Work - moderately heavy work

Level 3 Competitive and recreational sports - swimming, walking in rough forest terrain

Work - light labor

Level 2 Work - light work, walking on uneven ground

Level 1 Work - sedentary work, walking on even ground

Level 0 Sick leave or disability pension because of knee problems (c) (i) Open Access This article is licensed under a Creative Commons Attribution 4.0 International License, which permits use, sharing, adaptation, distribution and reproduction in any medium or format, as long as you give appropriate credit to the original author(s) and the source, provide a link to the Creative Commons license, and indicate if changes were made. The images or other third party material in this article are included in the article's Creative Commons license, unless indicated otherwise in a credit line to the material. If material is not included in the article's Creative Commons license and your intended use is not permitted by statutory regulation or exceeds the permitted use, you will need to obtain permission directly from the copyright holder. To view a copy of this license, visit http://creativecommons.org/licenses/ by/4.0/. 